\title{
Reappraisal of the Origins of the Polymodal Molecular Mass Distributions in the Formation of Poly(methylphenylsilylene) by the Wurtz Reductive-Coupling Reaction
}

\author{
Richard G. J ones,* Ulrich Budnik, Simon J . Holder, and \\ William K. C. Wong
}

Centre for Materials Research, Department of Chemistry, University of Kent, Canterbury CT2 7NH, U.K.

Received April 2, 1996; Revised Manuscript Received September 4, $1996^{\otimes}$

\begin{abstract}
Variations of yields and molecular weight parameters of poly(methylphenylsilylene) formed through sodium-mediated Wurtz reductive coupling of dichloromethylphenylsilane in toluene, xylene, diethyl ether, tetrahydrofuran, and diphenyl ether both in the presence and in the absence of 15-crown-5 and at different temperatures are described. The effects of various terminating reagents are also considered. The crown ether when it is present in the reaction mixture is shown to act as a phase transfer agent for the sodium and this is interpreted as having two effects. The first is to activate the alkali metal for reductive coupling, which at lower temperatures is a necessary condition for polymer formation. The other is to transport the alkali metal to points on the high molecular weight polymer chains where, as a prelude to backbiting, it induces a highly selective chain scission; this is explained by it occurring only at gauche defects in the otherwise rodlike all-trans sequences within the polymer in solution. The polymodal molecular weight distributions that are commonplace for polysilylenes are rationalized in terms of a competition between such activation and degradation processes.
\end{abstract}

\section{Introduction}

Polysilylenes, otherwise known as polysilanes, are linear chain polymers with backbones of catenated silicon atoms. ${ }^{1}$ Although their formation was recognized more than 70 years ago, ${ }^{2,3}$ systematic studies only began about 20 years ago with the preparation of the first soluble homopolymers ${ }^{4,5}$ and copolymers. ${ }^{6,7}$ The field has since seen a great deal of activity ${ }^{8}$ and a large number of high molecular weight polysilylenes have now been prepared and characterized. They have interesting electronic properties arising from extensive $\sigma$-conjugation along the backbone and they have potential applications as photoresists, ${ }^{9,10}$ radical photoinitiators, ${ }^{11,12}$ pre-ceramic materials, 13,14 photoconductors, ${ }^{15}$ and nonlinear optical materials. ${ }^{16}$

Polysilylenes can be prepared by several synthetic routes including the dehydrogenative coupling of primary silanes, ${ }^{17}$ the anionic polymerization of masked disilylenes, ${ }^{18}$ and the ring opening polymerization of strained cyclosilanes. ${ }^{19}$ However, in spite of considerable effort to displace it, the Wurtz reductive-dechlorination of dichloroorganosilanes, shown in Scheme 1, remains the most general and common procedure for their synthesis.

Studies of the effects of a number of variables in the Wurtz reductive-coupling reaction have shown that the yields and molecular weight distributions of the product polymers can be greatly influenced by solvent effects, ${ }^{20-22}$ the nature of the metal, ${ }^{23-25}$ the active surface of metal, ${ }^{26,27}$ reaction temperatures, ${ }^{21,28,29}$ the addition of phase transfer catalysts, $,, 28,30$ and even the rate and order of addition of the dichlorosilane.5,21 Depending on the nature of the substituents and the specific reaction conditions, the molecular weights of the products vary from a few thousand to several million. To date there is no satisfactory and unifying mechanistic theory that can be used to determine a best procedure

* Author to whom correspondence should be addressed. 1996.

${ }^{\otimes}$ Abstract published in Advance ACS Abstracts, November 1 ,
Scheme 1

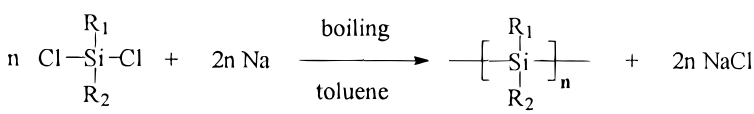

and the reaction conditions must be optimized for each polymerization. However, in searching for the best reaction conditions one faces the dilemma that each variable has its own marked effect on the polymerization and therefore can only be considered as a single contributory influence. For example, an investigation of the effect of the reaction temperature in a given solvent on the polymerization of a particular monomer requires all the other parameters mentioned above to be maintained constant. The best result that is then obtained is only the best temperature for that particular parameter set. Separate investigations for each reaction variable would not therefore necessarily lead to the best procedure, but by assessing the trends, reaction conditions close to the optimum might be achieved.

Usually the reaction is conducted in a boiling aromatic solvent such as toluene. Ethers or alkanes with boiling points sufficiently high as to maintain the metal in its molten state have also been used. Hence, the metal is readily, if not uniformly, distributed throughout the liquid phase. As indicated above, mechanism remains a contentious issue but because high molecular weight polymer is found at low monomer conversions it is the generally accepted belief ${ }^{31}$ that the very complex, heterogeneous process resembles more closely a chain growth rather than a step growth polymerization. The simplest model of the reaction ${ }^{21,32}$ is one in which polymer grows on the surface of the alkali metal following a slow initiation reaction. The ease with which the dichlorosilane reagent can approach the growing chain ends at the metal solution interface is one feature that influences the molecular weight of the polymer that is formed. This is determined by the extent of the solvation of the polymer chains and the alkali metal halide coproduct. However, the point has been made 29 that as the reaction proceeds the metal is consumed and the surface area decreases. Polymer 


\section{Scheme 2}

Initiation

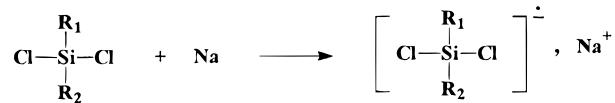

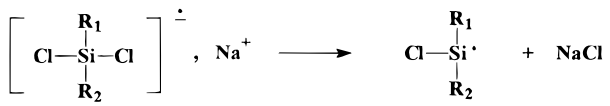

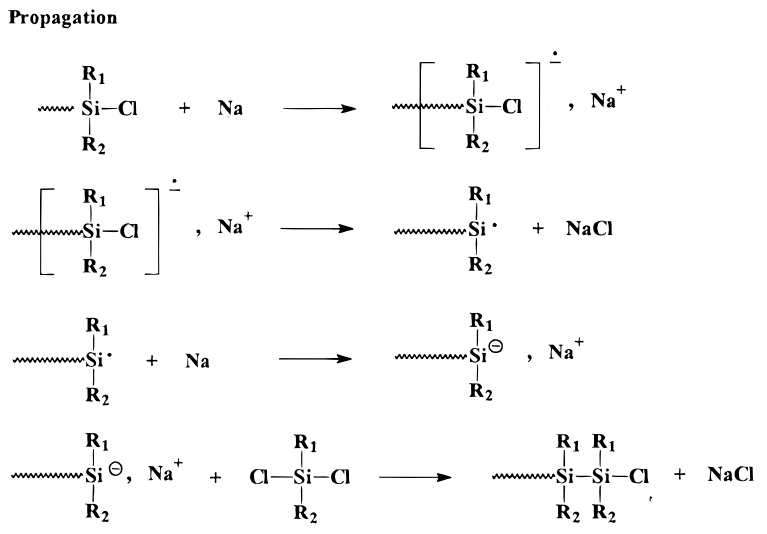

Termination

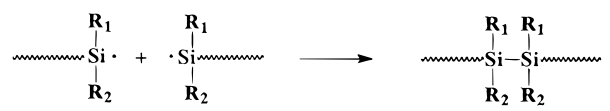

molecules are therefore continuously being forced to leave the metal surface. Early in the reaction these molecules must enter solution but in the later stages of reaction, if and when the solution phase is saturated, they precipitate, along with those growing molecules that still adhere to the surface. These effects al one were formerly thought to be sufficient to explain the broad molecular weight distributions that are characteristic of these syntheses.

Any full discussion of the effect of solvents, temperature, etc. must consider mechanism. In attempts to rationalize the formation of polysilylenes through Wurtztype polymerization, various intermediates have been suggested. These include silyl radicals, radical anions, anions and even silylenes. An anionic chain mechanism proposed by Gauthier and Worsfold ${ }^{9}$ was earlier shown ${ }^{33}$ to give a basis for an explanation of the observed polymodal molecular weight distributions. However, despite EPR studies ${ }^{34}$ offering no evidence for radical intermediates, the body of available mechanistic evidence does point to their involvement and it is virtually impossible to propose a mechanism from which they are excluded. Scheme 2 depicts a modification of the Gauthier and Worsfold chain mechanism that has been proposed by Matyjaszewski, ${ }^{31}$ in which the propagation is sustained by a sequence of four reactions involving all of the intermediates listed above, with the exception of silylenes. Though each would have a different lifetime and one would have the longest, it is reasonable to assume that at any instant, all would be present in a reaction mixture and ther efore amenable to trapping studies. Some such studies ${ }^{1}$ have been conducted but it is difficult to reach conclusions that are unambiguous.

Matyjaszewski et al. ${ }^{27,35}$ and Miller et al. ${ }^{28,36,37}$ have previously reported a modification of the synthesis in which the reactions were conducted in toluene at temperatures far below the melting point of sodium. They reported very high molecular weight poly(methylphenylsilylene) (PMPS) with monomodal molecular weight distributions. The yields were lower than they would be under boiling conditions so it became clear that the polymerization temperature is apparently critical in the heterogeneous Wurtz-type polymerization process. This was underlined when we described a low temperature synthesis of PMPS in diethyl ether. ${ }^{29}$ Tolerably high molecular weight PMPS was obtained in good yield but for this modification to be effective it was found necessary to include the phase transfer catalyst 15-crown-5 in the reaction mixture. The effect of this addition has never been addressed in the context of the accepted mechanism ${ }^{31}$ and the solubility considerations as the nature of the solvent medium and the reaction temperatures are varied. Nor has the significance of the reaction conducted at low temperatures using homogeneous reagents ${ }^{38,39}$ been discussed in the context of understanding the reaction as it is usually conducted using heterogeneous reagents. This paper addresses these aspects. We describe here our detailed studies of the Wurtz reductive-coupling procedure for the synthesis of PMPS as a function of widely varying reaction conditions and we offer a new explanation of the origins of the polymodal molecular mass distributions that are an unavoidable characteristic of the polymer formed using heterogeneous reagents.

\section{Experimental Section}

Materials. Prior to use, toluene, xylene, and diphenyl ether were distilled from sodium, and diethyl ether and tetrahydrofuran (THF) were distilled from sodium/potassium alloy. Dichloromethylphenylsilane (Lancaster Synthesis) was also distilled before use and stored over magnesium turnings and 4A molecular sieves. 1,4,7,10,13-pentaoxacyclopentadecane (15-crown-5) was used as supplied by Lancaster. Sodium (Fisons) was stored under paraffin oil and before use it was cut and washed under toluene.

All glassware was flame dried. Sodium dispersions for use at temperatures bel ow the melting point $\left(98^{\circ} \mathrm{C}\right)$ were prepared immediately prior to use by melting the freshly cut metal in mineral oil before dispersing it into a fine sand through the use of an homogenizer (UItra-Turrax T 8, IKA Labortechnik, Staufen). The oil was removed by washing three times with diethyl ether. All vol atiles were then removed under vacuum before the solvent of reaction was added. All other procedures have been described previously. ${ }^{29}$

Apparatus and Procedures. Polymer structures were characterised as PMPS using NMR spectroscopy (J EOL GX270), ultraviolet-visible spectroscopy (Shimadzu UV-240), and FTIR spectroscopy (ATI Mattson). Molecular weights of the polymers were measured as linear polystyrene equivalents in tetrahydrofuran solution using size exclusion chromatography equipment supplied by Polymer Laboratories Ltd. and equipped with a mixed bed $5 \mathrm{~mm}$ PLgel column. The calibration was over the molecular weight range 162 to $1.03 \times 10^{6}$. Relative amounts of different fractions were determined from the integrated molecular weight distribution curves.

General Procedure for the Polymerization of Dichloromethylphenylsilane. All polymers were prepared using the same general procedure as described here for the preparation of PMPS in diethyl ether with the addition of 15-crown5: To a two-necked round bottom flask ( $100 \mathrm{~mL}$ ) equipped with an egg-shaped PTFE $(25 \times 12 \mathrm{~mm})$ stirring bar and a condenser was added freshly cut sodium metal $(0.61 \mathrm{~g}, 0.0265 \mathrm{~mol})$ under a nitrogen atmosphere using Schlenk-line techniques. The sodium was transformed to a fine dispersion as outlined above. Tothe sodium dispersion was added diethyl ether $(30 \mathrm{~mL})$ and 15 -crown-5 $(0.5 \mathrm{~mL}, 0.0027 \mathrm{~mol})$. After rapid addition of dichl or omethyl phenylsilane (DCMPS) $(2 \mathrm{~mL}, 0.0120 \mathrm{~mol})$ the reaction mixture was rapidly stirred under reflux for $2 \mathrm{~h},{ }^{40}$ after which the mixture was allowed to cool to room temperature. While cooling in an ice bath, rapid stirring was maintained and methanol ( $15 \mathrm{~mL}$ ) was added cautiously to quench any unreacted sodium. A further $1 \mathrm{~h}$ of stirring at room temperature all owed for complete termination. At this 
point $40 \mathrm{~mL}$ of water was added. The organic layer was then decanted and added to stirred methanol $(250 \mathrm{~mL})$ at room temperature. The precipitated polymer was collected by filtration, washed with methanol, and dried in a vacuum oven. It contained a very small fraction that was insoluble in solvents such as THF and chloroform.

If not so stated, the data cited in the tables that follow pertain to the crude polymers. Otherwise, $0.6 \mathrm{~g}(5.0 \mathrm{mmol})$ of the crude polymer was extracted in a Soxhlet apparatus with $150 \mathrm{~mL}$ of hexane for $24 \mathrm{~h}$. The extracted polymer was dried in a vacuum oven. Following the evaporation of the hexane the extract residues were dissolved in a minimum of toluene and added to stirred methanol $(150 \mathrm{~mL})$. The oligomeric and polymeric material that was precipitated in this way was collected by filtration, washed with methanol, and dried in a vacuum oven.

Typical analytical data after extraction of the polymer with hexane to remove oligomers and reprecipitation in methanol from a filtered toluene solution are as follows: IR (film): $v / \mathrm{cm}^{-1}$ $=3082$, 3066, 3045, 3024, 3011, 2957, 2895, 1427, 1408, 1257, $1246,1098,781,753,728,697$. UV $\left(\mathrm{CH}_{2} \mathrm{Cl}_{2}\right): \lambda_{\max } / \mathrm{nm}(\log \epsilon)$

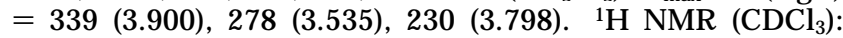
$\delta / p p m=-1.0-1.3\left(\mathrm{br}, \mathrm{m}, 3 \mathrm{H}, \mathrm{CH}_{3}\right), 6.0-8.4(\mathrm{br}, \mathrm{m}, 5 \mathrm{H}$, aromatic $\mathrm{H}) .{ }^{13} \mathrm{C} \mathrm{NMR}\left(\mathrm{CDCl}_{3}\right): \delta / p p m=-6.3(\mathrm{br}), 127.1(\mathrm{br})$, 129.1 (br), 133.3 (br), 134.8 (br), $136.2(\mathrm{br}) .{ }^{29} \mathrm{Si}\left(\mathrm{CDCl}_{3}\right)$ : $\delta / p p m=-39.2,-39.9,-41.2 . \quad \mathrm{C}_{7} \mathrm{H}_{8} \mathrm{Si}$ (120.23): calcd. C 69.93 H 6.71 found C 69.21 H 6.89 .

\section{Results}

The present study is focused on solvent, additive, and temperature effects in the synthesis of PMPS. However, in order to establish the comparability of different systems, the optimum stoichiometric ratios of sodium/ DCMPS required for the reactions in boiling toluene and diethyl ether were sought. These solvents were chosen for the study because toluene is a good solvent of PMPS and the most common one used in the Wurtz-type synthesis of polysilylenes, while diethyl ether is a relatively poor solvent of PMPS but one which sustains a controlled synthesis at much lower temperatures when 15-crown-5 is included in the system.

Effect of Stoichiometry. A representative molecular weight distribution for PMPS formed through the reaction of $\mathrm{Na}$ with DCMPS in boiling toluene is shown in Figure 1a. As is generally to be expected for a polysilylene formed in the reaction of a dichlorosilane in refluxing toluene, ${ }^{1}$ the distribution is trimodal with three distinct regions: (A) $M_{r, w}<1000$ (oligomers), (B) $M_{r, w}=1000$ to $5 \times 10^{4}$, and (C) $M_{r, w}=5 \times 10^{4}$ to several million. Figure la also depicts the integrated distribution from which it can be seen that the ratio of low molecular weight $\left(L_{p}\right)$ to high molecular weight $\left(H_{p}\right)$ polymer is about 70:30. Table 1 lists yields and molecular weight parameters for a series of such reactions at different mole ratios of $\mathrm{Na}$ to DCMPS.

Increasing the amount of sodium leads to significantly improved yields of polymer approaching a limiting value of approximately $55 \%$. There is a corresponding steady increase of the very high molecular weight fraction (C) from about 3\% for the reaction in which DCMPS is in the greatest excess over sodium up to $30 \%$ for the reaction in which a $10 \%$ excess of sodium has been employed. A further increase in the mole ratio of $\mathrm{Na}$ to DCMPS leads to a steady decrease of the high molecular weight fraction and with sodium in twice stoichiometric ratio no high molecular weight polymer can be detected. These variations are reflected in the molecular weight parameters of the polymers, the maximum polydispersity being realized when the re agents are in stoichiometric ratio.

The corresponding results that were obtained for the reactions in refluxing diethyl ether are listed in Table
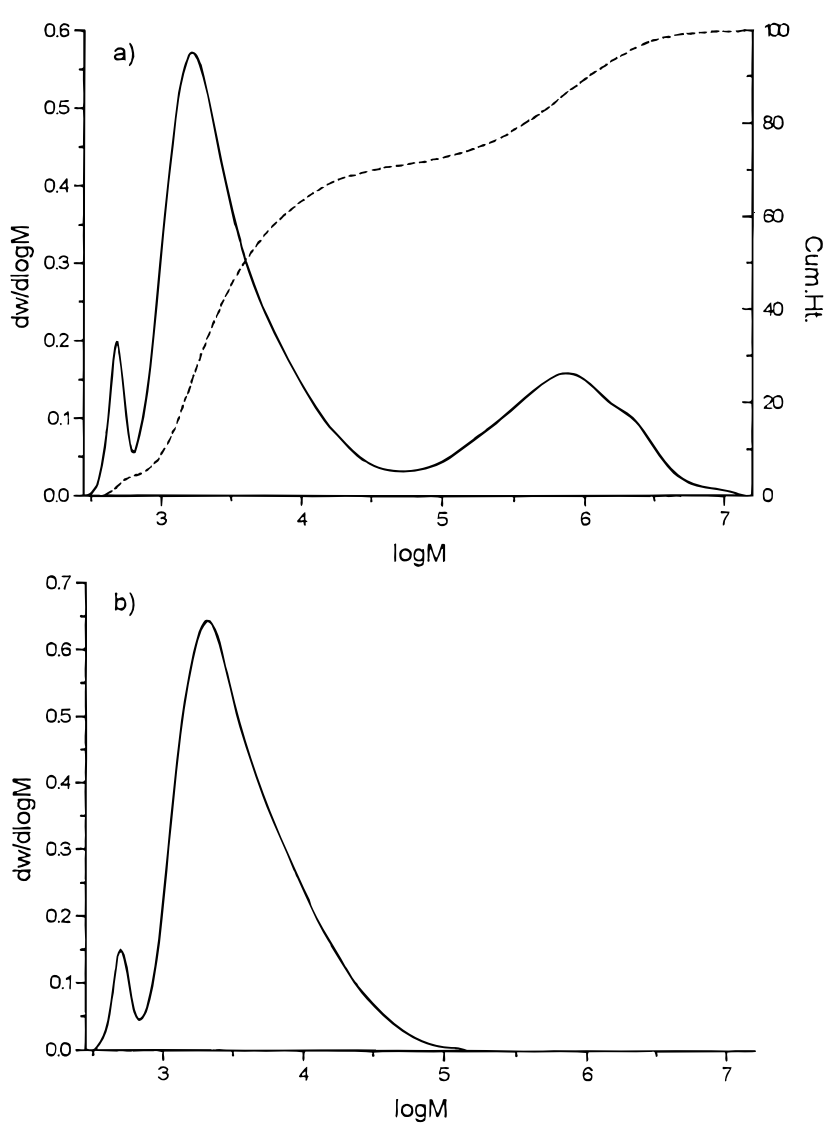

Figure 1. Representative molecular weight distributions of PMPS synthesized in refluxing toluene: (a) [Na]/[DCMPS] = 2.2 , $[15-$ crown -5$] /[\mathrm{Na}]=0 ;$ (b) $[\mathrm{Na}] /[\mathrm{DCMPS}]=2.2$, [15-crown$5] /[\mathrm{Na}]=0.1$.

Table 1. Influence of the Amount of Sodium on the Formation of PMPS in Toluene

\begin{tabular}{cccccl}
\hline \multirow{2}{*}{ solvent } & $\begin{array}{c}\text { mole ratio } \\
\text { Na:DCMPS }\end{array}$ & yield $(\%)$ & $\mathrm{M}_{\mathrm{n}}\left(\times 10^{3}\right)$ & $\mathrm{M}_{\mathrm{w}} / \mathrm{M}_{\mathrm{n}}$ & $\mathrm{L}_{\mathrm{p}} / \mathrm{H}_{\mathrm{p}}$ \\
\hline toluene & 0.4 & traces & 0.7 & 12 & $97 / 3$ \\
& 1.1 & 10 & 2.4 & 40 & $90 / 10$ \\
& 1.6 & 21 & 2.7 & 54 & $75 / 25$ \\
& 2.2 & 31 & 2.2 & 63 & $70 / 30$ \\
& 2.8 & 54 & 2.6 & 33 & $95 / 5$ \\
& 3.4 & 57 & 3.1 & 35 & $90 / 10$ \\
& 4.0 & 54 & 3.0 & 6.9 & 100
\end{tabular}

Table 2. Influence of the Amount of Sodium on the Formation of PMPS in Diethyl Ether

\begin{tabular}{ccccc}
\hline solvent & $\begin{array}{c}\text { mole ratio } \\
\text { Na:DCMPS }\end{array}$ & yield $(\%)$ & $\mathrm{M}_{\mathrm{n}}\left(\times 10^{3}\right)$ & $\mathrm{M}_{\mathrm{w}} / \mathrm{M}_{\mathrm{n}}$ \\
\hline diethyl ether & 1.1 & traces & 1.8 & 1.6 \\
& 1.6 & 3 & 2.0 & 3.4 \\
& 2.2 & 8 & 1.9 & 3.8 \\
& 2.8 & 19 & 2.0 & 4.5 \\
& 3.4 & 22 & 2.1 & 5.6 \\
& 4.0 & 35 & 3.5 & 3.0 \\
& 6.0 & 24 & 2.6 & 4.3
\end{tabular}

2 and a typical size exclusion chromatogram is shown in Figure 2a.

The most obvious differences between the reactions in toluene and in diethyl ether is the absence of the formation of any significant high molecular weight fraction and the lesser yields of polymer in the ether system. Discounting the oligomeric fraction which is easi ly removed by hexane extraction, all of the pol ymers from the diethyl ether system were found to have almost monomodal molecular weight distributions and any high molecular weight tail never exceeded about $3 \%$ of the overall yield. Increasing the amount of sodium again 

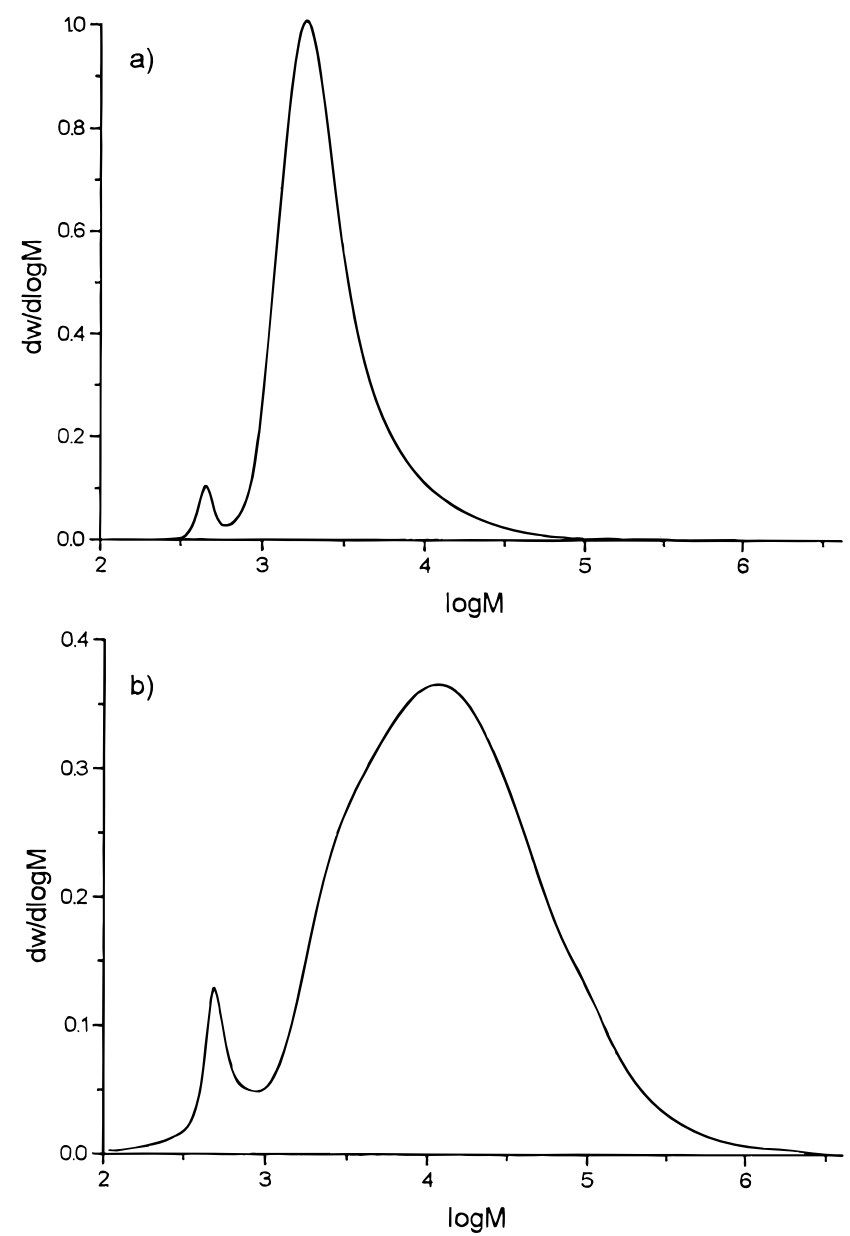

Figure 2. Representative molecular weight distributions of PMPS synthesized in refluxing diethyl ether: (a) [Na]/ $[$ DCMPS $]=2.2$, [15-crown-5]/[Na] = 0; (b) $[\mathrm{Na}] /[\mathrm{DCMPS}]=$ 2.2, $[15$-crown-5]/[Na] $=0.1$.

leads to significantly improved yields. In the reaction with the greatest excess of DCMPS over sodium only traces of polymer were isolated but with increasing proportion of sodium the yields steadily increase to a maximum of $35 \%$ at twice the stoichiometric ratio of sodium to DCMPS. Thereafter the yields fall back to about $25 \%$. The mol ecular weight parameters follow a similar pattern.

Effect of Added 15-Crown-5. Although from refluxing toluene reasonable amounts of high molecular weight polymer can be obtained, the molecular weight distributions are very broad. On the other hand, the polymers obtained from reactions in refluxing diethyl ether were found to have almost monomodal molecular weight distributions but with yields and molecular weights that might be considered to be too low to be useful in applications. Recently, it has been reported ${ }^{9,33,37}$ that the incorporation of catalytic amounts of crown ether in the reactions in toluene leads to polymers whose mol ecular weight distributions tend to the monomodal, albeit at the expense of molecular weight. In contrast, the inclusion of 15-crown-5 in the reactions in diethyl ether resulted in a striking increase of yields and molecular weights. ${ }^{29,33}$ Whereas for the reaction in toluene it has been reported ${ }^{37}$ that the best results were obtained by using only catalytic amounts of 15-crown-5, although the term "best" was never specified, any information about the optimum amount needed in other solvents has been sparse. Therefore, the influence of varying concentrations of 15 -crown-5 on the polymerizations of DCMPS in refluxing toluene and refluxing diethyl ether has also been investigated.

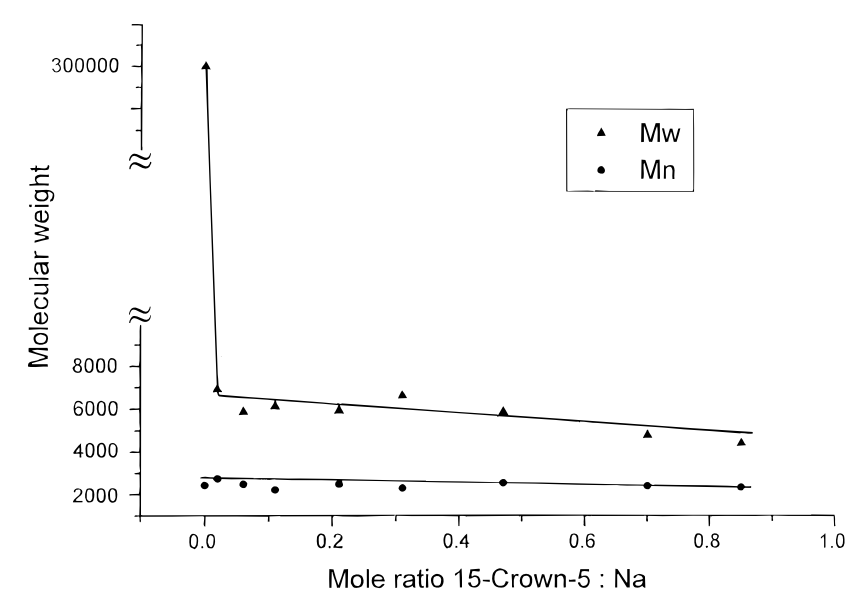

Figure 3. Variation with 15-crown-5 concentration of the weight-average and number-average molecular weights of PMPSs synthesized in refluxing toluene, [Na]/[DCMPS] = 2.2.

Table 3. Influence of the Amount of 15-Crown-5 on the Formation of PMPS in Toluene, [Na]/[DCMPS] = 2.2

\begin{tabular}{ccccc}
\hline \multirow{2}{*}{ solvent } & $\begin{array}{c}\text { mole ratio } \\
\text { Na:15-crown-5 }\end{array}$ & yield (\%) & $\mathrm{M}_{\mathrm{n}}$ & $\mathrm{M}_{\mathrm{w}} / \mathrm{M}_{\mathrm{n}}$ \\
\hline toluene & 0.00 & 48 & 2.4 & 133 \\
& 0.02 & 39 & 2.8 & 2.5 \\
& 0.06 & 33 & 2.5 & 2.4 \\
& 0.11 & 31 & 2.2 & 2.8 \\
& 0.21 & 23 & 2.5 & 2.4 \\
& 0.30 & 21 & 2.3 & 2.9 \\
& 0.47 & 19 & 2.3 & 2.3 \\
& 0.70 & 19 & 2.4 & 2.0 \\
& 0.85 & 17 & 2.3 & 1.9
\end{tabular}

Table 4. Influence of the Amount of Sodium on the Formation of PMPS in Toluene, [15-Crown-5]/[Na] = 0.1

\begin{tabular}{ccccc}
\hline \multirow{2}{*}{ solvent } & $\begin{array}{c}\text { moleratio } \\
\text { Na:DCMPS }\end{array}$ & yield $(\%)$ & $\mathrm{M}_{\mathrm{n}}\left(\times 10^{3}\right)$ & $\mathrm{M}_{\mathrm{w}} / \mathrm{M}_{\mathrm{n}}$ \\
\hline toluene & 1.1 & 20 & 3.1 & 2.2 \\
& 1.6 & 19 & 2.5 & 2.3 \\
& 2.2 & 31 & 2.2 & 2.8 \\
& 2.8 & 30 & 2.1 & 2.3 \\
& 3.4 & 20 & 2.5 & 2.3 \\
& 4.0 & 18 & 2.8 & 2.1
\end{tabular}

The results obtained for the reactions in refluxing toluene are shown in Figure 3 and Tables 3 and 4, and for purposes of comparison a typical size exclusion chromatogram of one of the product polymers is depicted in Figure $1 \mathrm{~b}$ alongside that of the corresponding reaction in the absence of crown ether. The addition of 15crown-5 has a marked effect on the molecular weights and molecular weight distributions of the product polymers. Over the whole range of reaction mixture compositions the crude polymers were found to have almost monomodal and relatively narrow molecular weight distributions; only small amounts of oligomeric cyclosilanes perturbed the otherwise normal distributions. The $M_{w}$ values ranged between 4000 and 7000 and were therefore significantly lower than those for the polymer obtained from the systems without added crown ether. From Figure 3 and Table 3 it is evident that increasing concentrations of crown ether cause a progressive decrease in the values of the average molecular weight parameters but this is as nothing compared to the tremendous effect of the first addition of only a very small amount. Similarly, yields decreased steadily with increasing concentration of the crown ether. From Table 4, which represents the variation of yields and molecular weights with reagent stoichiometry at constant crown ether concentration, it is seen that maximum yiel ds of about $30 \%$ were obtained when the 


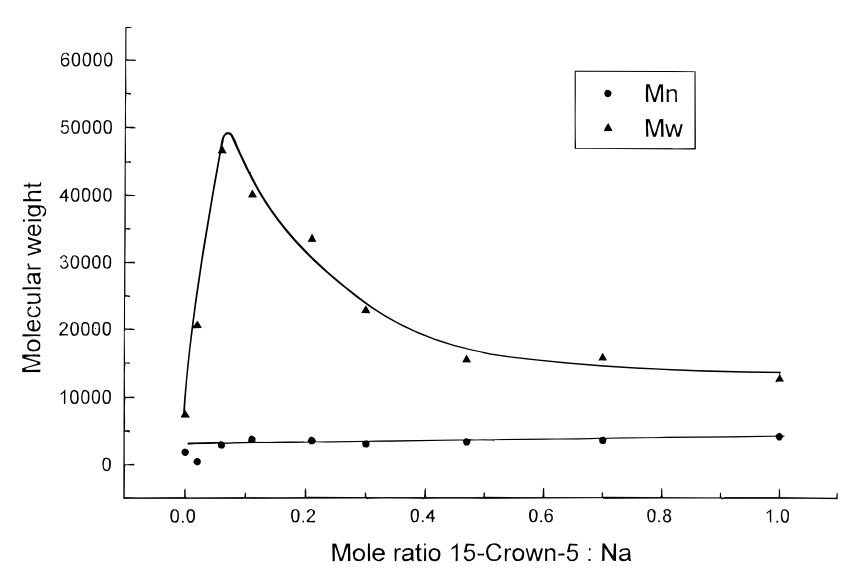

Figure 4. Variation with 15-crown-5 concentration of the weight-average and number-average molecular weights of PMPSs synthesized in refluxing diethyl ether, [ $\mathrm{Na}] /[\mathrm{DCMPS}]$ $=2.2$.

Table 5. Influence of the Amount of 15-Crown-5 on the Formation of PMPS in Diethyl Ether, [Na]/[DCMPS] $=\mathbf{2 . 2}$

\begin{tabular}{ccccc}
\hline solvent & $\begin{array}{c}\text { mole ratio } \\
\text { 15-crown-5:Na }\end{array}$ & yield (\%) & $\mathrm{M}_{\mathrm{n}}\left(\times 10^{3}\right)$ & $\mathrm{M}_{\mathrm{w}} / \mathrm{M}_{\mathrm{n}}$ \\
\hline diethyl ether & 0.00 & 8 & 1.9 & 3.8 \\
& 0.02 & 85 & 2.1 & 9.8 \\
& 0.06 & 92 & 3.0 & 15.7 \\
& 0.11 & 67 & 3.8 & 10.7 \\
& 0.21 & 76 & 3.6 & 9.4 \\
& 0.30 & 73 & 3.1 & 7.4 \\
& 0.47 & 63 & 3.4 & 4.6 \\
& 0.70 & 63 & 3.6 & 4.4 \\
1.00 & 61 & 4.1 & 3.1
\end{tabular}

Table 6. Influence of the Amount of Sodium on the Formation of PMPS in Diethyl Ether, [15-Crown-5]/[Na] $=0.1$

\begin{tabular}{ccccc}
\hline \multirow{2}{*}{ solvent } & $\begin{array}{c}\text { mole ratio } \\
\text { Na:DCMPS }\end{array}$ & yield $(\%)$ & $\mathrm{M}_{\mathrm{n}}\left(\times 10^{3}\right)$ & $\mathrm{M}_{\mathrm{w}} / \mathrm{M}_{\mathrm{n}}$ \\
\hline diethyl ether & 1.1 & 32 & 2.8 & 6.4 \\
& 1.6 & 50 & 2.8 & 11 \\
& 2.2 & 68 & 3.6 & 12 \\
& 2.8 & 66 & 4.2 & 9.2 \\
& 4.0 & \multicolumn{3}{c}{ no polymer found }
\end{tabular}

reagents were in stoichiometric ratio but these are not significantly greater than those of about $20 \%$ obtained at other ratios.

In contrast are the results obtained for the corresponding reactions in refluxing diethyl ether which are shown in Figure 4 and Tables 5 and 6, and again for the purposes of comparison, a typical size exclusion chromatogram of one of the polymers is shown in Figure 2b. As can be seen from Figure 4 and Table 5, following an initial sharp rise in yields and molecular weights of the products, both are progressively reduced by increasing concentrations of the crown ether. However, from Table 6 it is clear that over the whole range of reaction mixture compositions the polymers were found to have significantly higher molecular weights and to be obtained in much greater yields than for the corresponding reactions in refluxing diethyl ether without any 15crown-5. In the presence of the crown ether, the polymer molecular weight distributions become bi modal and the polydispersities are correspondingly greater.

From Figures 3 and 4 it is evident that only catalytic amounts of the crown ether are required to produce the maximum effect, the most dramatic changes having been achieved at molar ratios of crown ether to sodium of less than 1:10. In the toluene systems the effect is to destroy the high molecular weight fraction. This is in complete contrast with the ether systems in which

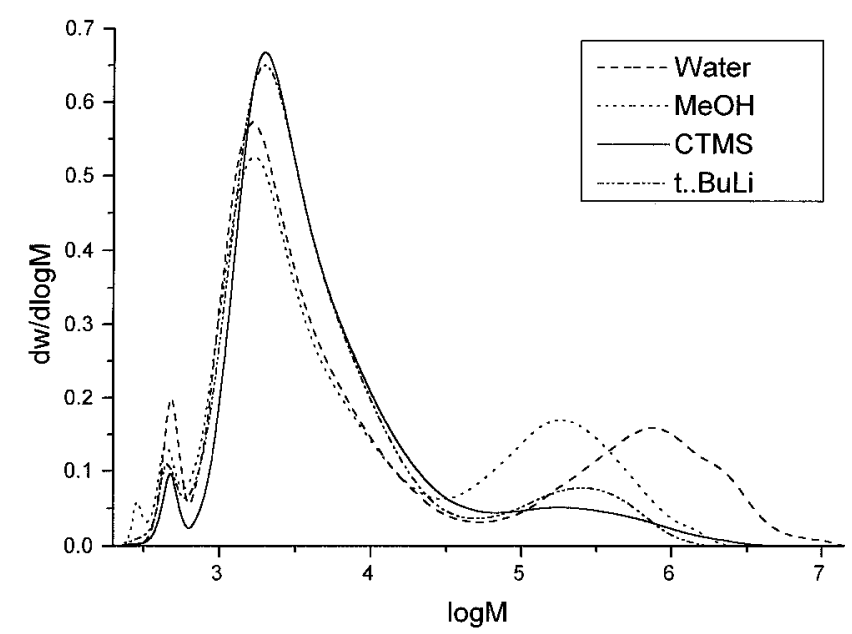

Figure 5. Comparison of the molecular weight distributions of PMPSs isolated from refluxing toluene following termination by water, methanol, tert-butyllithium, and chlorotrimethylsilane.

the effect is a pronounced extension of the product molecular weight distributions toward higher molecular weights.

Effect of Different Terminating Agents. It was reasoned that different terminating agents might influence yields, molecular weights, and molecular weight distributions and so distort any conclusions that might be reached from studies such as the foregoing. Thus, the effects of using water, methanol, tert-butyllithium (t-BuLi), and chlorotrimethylsilane (CTMS) as different terminating agents for active chain ends were investigated for reactions (a) in toluene and (b) in diethyl ether in the presence of 15-crown-5. Because these particular systems provide medium to high molecular weight polymer, the effects, if any, of the different terminating agents on the molecular weights and distributions were expected to be pronounced. In this study, water and methanol were added in large excess in the terminating procedures as they are capable of serving the dual purpose of terminating all of the chain ends whether they be in the silyl anionic or silyl chloride forms (see Scheme 2), and of destroying any alkali metal that might remain. In contrast, the t-BuLi was expected to terminate only $\mathrm{Si}-\mathrm{Cl}$ chain ends and the CTMS was considered to be only a good terminating agent for silyl anionic chains ends. Nonetheless, t-BuLi would also react with unconsumed monomer and CTMS with unconsumed sodium so if they were added in sufficient quantity the polymerizations would again be effectively terminated. With a very considerable margin allowed for error, it was known from previous experience that a conversion of at least $60 \%$ of the original DCMPS had been achieved within the chosen reaction time of $2 \mathrm{~h}$ in either polymerizing system. It was on this basis that the calculation of sufficient quantities of either t-BuLi or CTMS was made. After allowing time for the reagent to react, $30 \mathrm{~mL}$ of $\mathrm{MeOH}$ was then added before the polymers were isolated using the standard procedure. The results are depicted in Figures 5 and 6 .

Water, methanol, and CTMS show little difference in their effect on the reaction conducted in diethyl ether but the use of t-BuLi as a terminating agent appears to give a slightly narrower molecular weight distribution. In contrast, for the reactions in toluene, when compared to the systems terminated with either water or methanol, the t-BuLi and CTMS seem to be equally effective in enhancing the yields of the lower molecular weight fraction while suppressing the formation of higher 


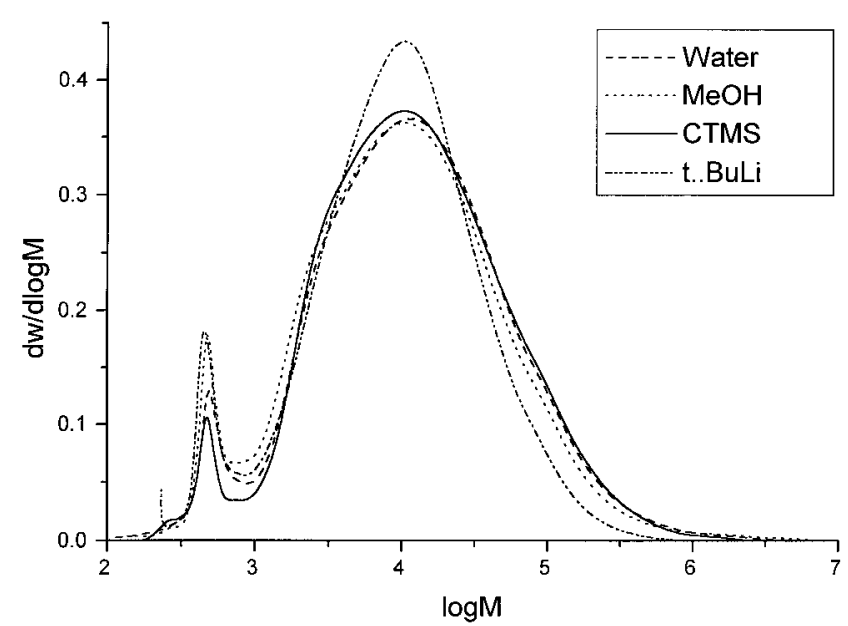

Figure 6. Comparison of the molecular weight distributions of PMPSs isolated from refluxing diethyl ether following termination by water, methanol, tert-butyllithium, and chlorotrimethylsilane.

Table 7. Influence of the Solvent on the Formation of PMPS by the Wurtz Coupling Reaction, [Na]/[DCMPS] = 2.2

\begin{tabular}{|c|c|c|c|c|c|c|}
\hline \multirow[b]{2}{*}{ solvent } & \multicolumn{2}{|r|}{ reaction } & \multirow{2}{*}{$\begin{array}{l}\text { poly- } \\
\text { mera }\end{array}$} & \multirow{2}{*}{$\begin{array}{c}\text { yield } \\
(\%)\end{array}$} & \multirow{2}{*}{$\begin{array}{c}M_{n} \\
\left(\times 10^{3}\right) \\
\end{array}$} & \multirow{2}{*}{$\begin{array}{l}M_{w} / \\
M_{n}\end{array}$} \\
\hline & time & temperature & & & & \\
\hline \multirow[t]{3}{*}{ diethyl ether } & 2 & reflux & $a$ & 8 & 1.9 & 3.8 \\
\hline & & & b & 2 & 4.1 & 6.8 \\
\hline & & & C & 4 & 2.2 & 1.2 \\
\hline \multirow{3}{*}{ tetrahydrofuran } & 2 & reflux & a & 53 & 5.6 & 24 \\
\hline & & & $\mathrm{b}$ & 47 & 13 & 15 \\
\hline & & & c & 3 & 3.1 & 1.3 \\
\hline \multirow[t]{3}{*}{ diphenyl ether } & 0.5 & $195 \pm 5^{\circ} \mathrm{C}$ & a & 65 & 2.6 & 2.1 \\
\hline & & & b & 33 & 4.7 & 1.7 \\
\hline & & & c & 31 & 2.3 & 1.2 \\
\hline \multirow{3}{*}{ toluene } & 2 & reflux & a & 48 & 2.4 & 133 \\
\hline & & & b & 24 & 11 & 50 \\
\hline & & & C & 19 & 2.1 & 1.3 \\
\hline \multirow[t]{3}{*}{ xylene } & 2 & reflux & a & 42 & 2.0 & 5.4 \\
\hline & & & b & 16 & 4.9 & 8.1 \\
\hline & & & c & 22 & 2.1 & 1.3 \\
\hline
\end{tabular}

a a, crude polymer; $b$, polymer after $24 \mathrm{~h}$ extraction with hexane in a Soxhlet apparatus; c, polymer recovered from hexane after reprecipitation in methanol.

molecular weight polymer. On the other hand, the use of water as a terminating agent extends the high molecular weight fraction to even higher molecular weights. This is attributed to water being diprotic and thus able to join appropriately functionalized polymer molecules through the formation of siloxy linkages (vide infra). The other apparent differences in the chromatograms are considered to be so slight as to fall within normal experimental variation. Significantly, however, the choice of terminating agent does not alter the modality of the molecular weight distributions. Accordingly, for convenience in the product isolation procedures of subsequent polymerizations, termination with water was adopted as the standard practice.

Solvent and Temperature Effects. To extend understanding of the above effects the influence of five different solvents (diethyl ether, tetrahydrofuran (THF), diphenyl ether, toluene, and xylene) on the formation of PMPS were compared over a range of temperatures both in the presence and absence of 15-crown-5. In the first instance, all of the polymerizations were conducted at the boiling temperatures of the solvents in the absence of crown ether. ${ }^{41}$ A $10 \%$ stoichiometric excess of sodium was used in every case. The results displayed in Table 7 show the yields and molecular weights of (a) the crude polymers, (b) the polymer remaining after
Table 8. Influence of the Solvent on the Formation of PMPS by the Wartz Coupling Reaction, [Na]/[DCMPS] = 2.2, $[15-$ Crown-5]/[Na] $=0.1$

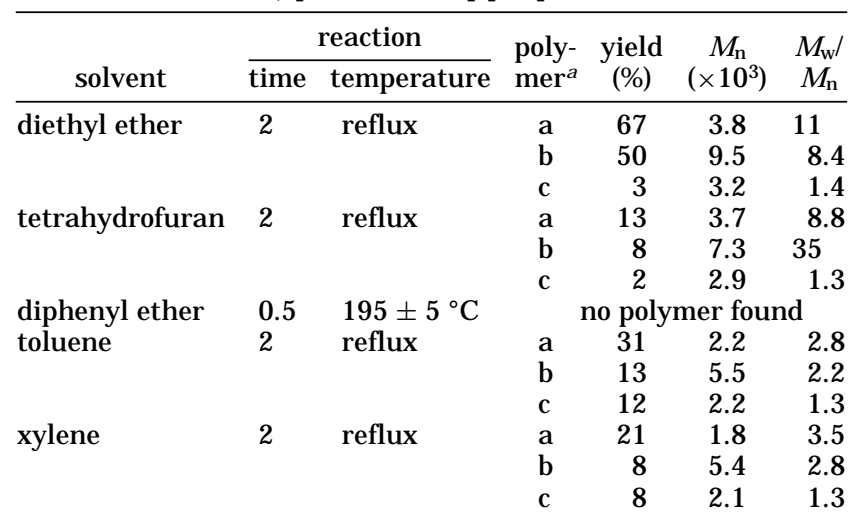

a a, crude polymer; $b$, polymer after $24 \mathrm{~h}$ extraction with hexane in a Soxhlet apparatus; $c$, polymer recovered from hexane after reprecipitation in methanol.

extraction with hexane for $24 \mathrm{~h}$ in a Soxhlet apparatus, and (c) the low molecular weight fraction recovered from the hexane by reprecipitation in methanol. The results for the corresponding reactions carried out in the presence of 15 -crown- 5 in $1 / 10$ mole ratio to sodium are shown in Table 8.

As evidenced by ${ }^{29} \mathrm{Si}$ NMR measurements, $24 \mathrm{~h}$ of Soxhlet extraction of the crude product with hexane removes a very small amount of a low molecular weight material containing siloxy linkages, the ubiquitous low molecular weight cyclosilanes, and a narrow distribution oligosilane fraction with a molecular weight of about 2000-3000. There is no evidence of residual siloxy linkages within the extracted polymers, and their ${ }^{29} \mathrm{Si}$ NMR spectra display only those resonances expected for PMPS main chain atoms ${ }^{42}$ and end groups. The amount of polysilylene that could be recovered from the hexane fraction by reprecipitation in methanol is a measure of the amount of low molecular weight polymer in the crude product that is originally isolated, i.e., the fraction identified above as B in the overall distribution.

While acknowl edging that there is a temperature as well as a solvent effect to be considered, it is apparent that for the systems polymerizing under reflux, only the yield of the product from the diethyl ether system can be improved by including 15-crown-5. In all other cases the yiel d is significantly reduced by its inclusion and it seems to completely inhibit polymer formation in the diphenyl ether system.

The effect of temperature on the synthesis of PMPS in the various solvents is shown in Table 9. Where no figures are shown in the final column of this table, the systems did not yield any high molecular weight polymer.

The effects are varied but by no means random, for there are identifiable trends. For the reactions in toluene and xylene without added crown ether the yields steadily fall with reducing temperature. There is also a significant alteration of the ratio of high to low molecular weight polymer, the higher molecular weight fraction being favored at lower temperatures. If crown ether is included in the same systems the yields are markedly enhanced at temperatures below the melting point of the sodium but as the temperatures are further reduced the yields again fall. As already noted, there is no high molecular weight fraction when crown ether is present. For the reaction in diphenyl ether the yields also fall with decreasing temperature and again there is a redistribution from the low to the high molecular 
Table 9. Influence of the Temperature on the Formation of PMPS, $[\mathrm{Na}] /[\mathrm{DCMPS}]=2.2,[15-\mathrm{Crown}-5] /[\mathrm{Na}]=0.1$

\begin{tabular}{|c|c|c|c|c|c|c|}
\hline solvent & $\begin{array}{l}\text { reaction } \\
\text { temp } \\
\left({ }^{\circ} \mathrm{C}\right)\end{array}$ & $\begin{array}{c}15- \\
\text { crown-5 }\end{array}$ & $\begin{array}{c}\text { yield } \\
(\%)\end{array}$ & $\begin{array}{c}M_{n} \\
\left(\times 10^{3}\right)\end{array}$ & $\begin{array}{c}\mathrm{M}_{\mathrm{w}} / \\
\mathrm{M}_{\mathrm{n}}\end{array}$ & $\begin{array}{l}\mathrm{L}_{\mathrm{p}} / \\
\mathrm{H}_{\mathrm{p}}\end{array}$ \\
\hline \multirow[t]{4}{*}{ diethyl ether } & reflux & & 8 & 1.9 & 3.8 & \\
\hline & 0 & & 3 & 1.7 & 1.9 & \\
\hline & reflux & + & 67 & 3.8 & 11 & \\
\hline & 20 & + & 39 & 3.4 & 5.1 & \\
\hline \multirow[t]{7}{*}{ tetrahydrofuran } & reflux & & 53 & 5.6 & 24 & \\
\hline & 50 & & 54 & 9.4 & 23 & \\
\hline & 20 & & 64 & 6.9 & 11 & \\
\hline & 0 & & 47 & 5.5 & 3.6 & \\
\hline & reflux & + & 13 & 3.7 & 8.8 & \\
\hline & 50 & + & 39 & 4.3 & 5.3 & \\
\hline & 20 & + & 64 & 5.5 & 6.8 & \\
\hline \multirow[t]{8}{*}{ diphenyl ether } & $195^{a}$ & & 65 & 2.6 & 2.1 & $100 / 0$ \\
\hline & 125 & & 39 & 2.2 & 4.5 & $95 / 5$ \\
\hline & 75 & & 12 & 5.1 & 35 & $30 / 70$ \\
\hline & 50 & & 5 & 5.7 & 311 & $30 / 70$ \\
\hline & 195 & + & & & & \\
\hline & 125 & + & \multirow{2}{*}{\multicolumn{4}{|c|}{ no polymer found }} \\
\hline & 75 & + & & & & \\
\hline & 50 & + & 8 & 3.3 & 2.0 & \\
\hline \multirow[t]{10}{*}{ toluene } & reflux & & 48 & 2.4 & 133 & $70 / 30$ \\
\hline & 75 & & 21 & 2.7 & 55 & $70 / 30$ \\
\hline & 50 & & 13 & 3.3 & 37 & $30 / 70$ \\
\hline & 20 & & 6 & 4.3 & 51 & $50 / 50$ \\
\hline & 0 & & 1 & 4.7 & 13 & $80 / 20$ \\
\hline & reflux & + & 31 & 2.2 & 2.8 & \\
\hline & 75 & + & 41 & 4.3 & 4.3 & \\
\hline & 50 & + & 52 & 4.3 & 4.3 & \\
\hline & 20 & + & 24 & 3.6 & 5.8 & \\
\hline & 0 & + & 8 & 3.6 & 2.9 & \\
\hline \multirow[t]{8}{*}{ xylene } & reflux & & 42 & 2.0 & 5.4 & $97 / 3$ \\
\hline & 75 & & 15 & 2.6 & 54 & $50 / 50$ \\
\hline & 50 & & 7 & 5.0 & 202 & $20 / 80$ \\
\hline & 20 & & 7 & 5.1 & 27 & $20 / 80$ \\
\hline & 0 & & \multicolumn{3}{|c|}{ no polymer found } & \\
\hline & reflux & + & 21 & 1.8 & 3.5 & \\
\hline & 75 & + & 44 & 3.8 & 4.4 & \\
\hline & 50 & + & 42 & 1.8 & 7.8 & \\
\hline
\end{tabular}

a Reaction time $30 \mathrm{~min}$.

weight fraction once the temperature is less than that of the melting point of sodium. However, except at the lowest temperature studied, no polymeric product could be isolated when crown ether was included in this system. What little polymer could be isolated at $50^{\circ} \mathrm{C}$ did not contain a high molecular weight fraction. The polymerizations that are notably different are those in THF. In the absence of crown ether the variation of yield with temperature is small. All the yields are quite high but there is a narrowing of the molecular weight distribution as the temperature is reduced. When the crown ether is included its effect is destructive of the polymer at the boiling point of the solvent and at the other temperatures it appears to serve little useful purpose. However, it is notable that the molecular weights are even further reduced and the distributions are again narrowed. The range over which the diethyl ether systems can be studied is restricted by the low boiling point of the solvent which is well removed from the melting point of sodium. However, whether or not the crown ether is present it would again appear that reducing the temperature of these systems al so results in decreased yields and narrower distributions.

Variation $L_{p} / H_{p}$ with Time. Much of the following arguments depend upon an understanding of the ratio of the yields of low to high molecular weight polymer isolated from the various systems. I deally this would follow from a knowledge of the variation of the yields of the two fractions with time. Experiments that would give this would require representative sampling of a multiphase system in which the sustaining solvent is

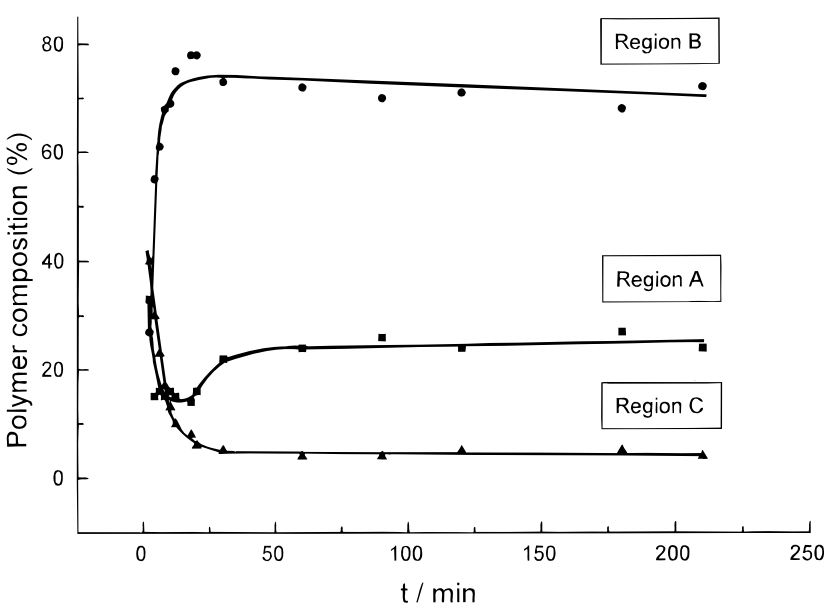

Figure 7. Variations with time of the proportions of the low $(\boldsymbol{\square})$, intermediate $(\boldsymbol{\bullet})$ and high ( $\mathbf{\Delta})$ molecular weight fractions of polymer isolated from the solution phase during a representative synthesis of PMPS in refluxing toluene.

usually boiling and this is so difficult as to be impractical. However, it has previously been shown that the overall reaction in diethyl ether follows a pseudo-firstorder rate law after an induction period ${ }^{29}$ and a similar variation has been observed for the sonically assisted synthesis in toluene at $60{ }^{\circ} \mathrm{C} .{ }^{27}$ Since toluene is a good solvent of the polymer it is reasoned that in this case representative variations of the product molecular weight distributions with time could be obtained by monitoring the polymer isolated from the solution phase and that the conclusions reached could be extended to the other systems by reasoning based on the foregoing results. Accordingly, a reaction vessel was equipped with a serum cap to allow small aliquots of the liquid phase to be withdrawn at regular intervals using a syringe. The syringe was equipped with a discharge filter to collect particulates that had inadvertently been withdrawn and the aliquots were then injected into methanol. Polymer was isolated in the usual way and Figure 7 depicts the results obtained for a reaction in boiling toluene using a small stoichiometric excess of sodium. In a complementary experiment it was shown that less than $5 \%$ of the polymer remained associated with the solid phase at the end of reaction.

It has previously been observed ${ }^{9}$ that a significant proportion of high molecular weight polymer is formed in the earliest stages of reaction and this result lends confirmation to that observation. However, it must be stressed that polymer found in the solution phase does not represent the whole product. For the diethyl ether system, a solvent in which the polymer is much less soluble, it is known that much of the product remains associated with the solid phase and that it is within this that the high molecular weight fraction is found. ${ }^{29}$ It must be assumed that the difference between the limiting value of $L_{p} / H_{p}$ observed in this experiment and the lower values reported above is because of a similar association, even in such a good solvent as toluene. Nonetheless, it is clear that formation of the high molecular weight fraction is favored in the earliest stages but as the reaction proceeds this is lost to the intermediate molecular weight fraction. Furthermore, with much the same time constant, there appears to be a small reduction in the proportion of cyclic oligomers that are found within the isolated polymer.

\section{Discussion}

We earlier explained the polymodal molecular weight distributions that arise during the synthesis of PMPS 


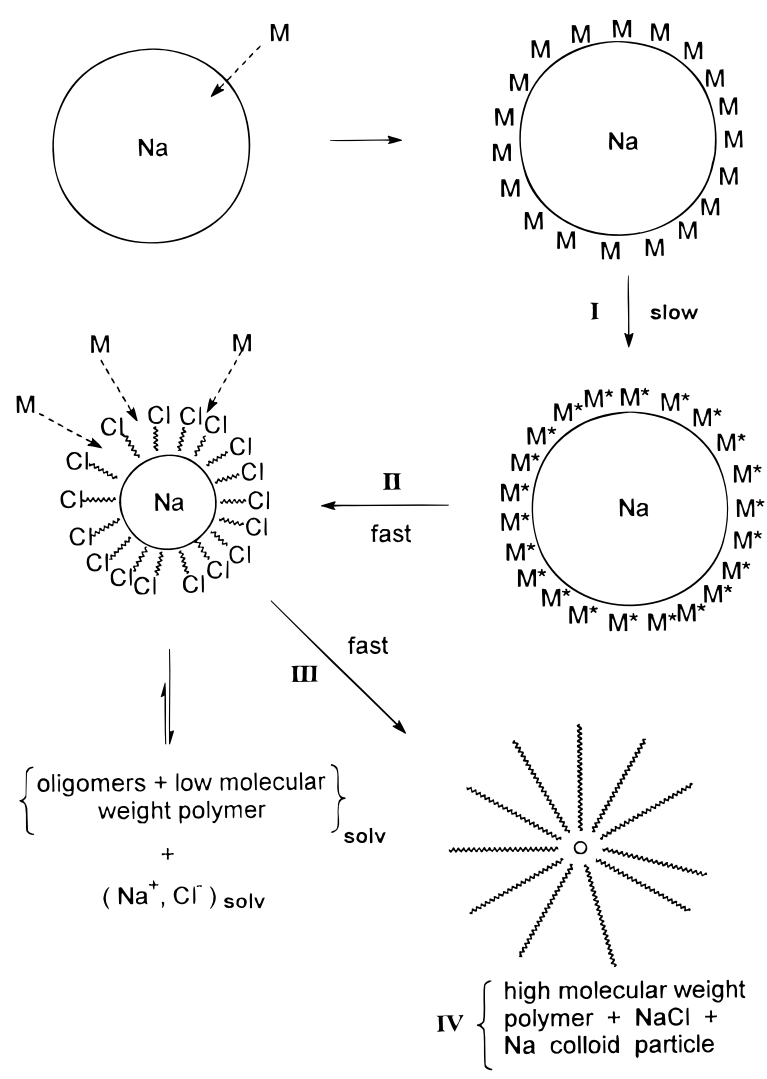

Figure 8. Schematic of the progress of an alkali metal mediated reductive coupling of a dihaloorganosilane in accordance with the processes described in ref 29.

through the sodium-mediated reductive coupling of DCMPS in diethyl ether as following solely from the heterogeneity of the system. ${ }^{29}$ By extension, we advanced the same ideas to explain the molecular weight distributions that result from the reaction in toluene and other solvents. The essential features are represented in Figure 8 in which (I) DCMPS is adsorbed onto the alkali metal surface where the polymerization undergoes a slow initiation process, (II) a rapid propagation ensues with the low molecular weight polymer formed in the early stages of reaction entering the as yet unsaturated solution as they are forced off the metal surface by the inevitable reduction in available surface area, (III) the solution phase is eventually saturated with polymer but the chain ends that remain associated with the surface continue to grow, and (IV) these polymer molecules precipitate among the solid mass of the alkali halide coproduct, a few remaining associated with the alkali metal particles which are reduced to only colloidal dimensions at the end of reaction. While not dismissing the idea that polymer solubility influences the reaction, this model is too simplistic to explain all the known observations of these complex polymerizations. However, stage IV of this model, the case for which is well supported by independent argument, 34 offers a ready explanation for the coupling of high molecular weight polymer molecules when reactive chain ends are destroyed by water rather than one of the alternative terminating agents used in this study. If all the active chain ends were in solution or trapped elsewhere within the solid phase, they would be well separated and their coupling by reaction with water would be most improbable. However, the stable colloidal alkali metal particles with which the remaining active centers are associated act as focal points for this reaction. When the colloidal alkali metal is destroyed by whatever agent, the active centers would then be in close proximity to each other in the presence of excess terminating agent. Methanol, t-BuLi, and CTMS would endcap the chains with methoxy, tert-butyl, and TMS groups, respectively, and the chain ends would then slowly diffuse away from each other. However, water molecules being diprotic would simultaneously terminate two chain ends and the necessary siloxy linkage would be established.

Accepting the above explanation of the significant shift to higher molecular weights of the high molecular fraction (region $\mathrm{C}$ of the typical distribution) of the polymer formed in toluene that is evident in Figure 6, it is significant that there is absolutely no indication from either Figure 5 or Figure 6 that the intermediate molecular weight fraction (region B) behaves in a similar way in either the toluene or diethyl ether systems. Thus, it must be concluded that very few, if any, of the polymer molecules in this fraction remain with their chain ends associated with colloidal alkali metal particles at the end of reaction. It follows that the formation of this fraction differs in some significant way from that of the high molecular weight fraction for it cannot simply be that molecules within this fraction just happen to be those that are discharged from the shrinking alkali metal surface either to solution or as precipitate. That would occur unsel ectively and would be expected to lead to a monomodal distribution extending over the whole molecular weight range.

In a discussion of the polymodal molecular weight distributions Matyjaszewski et al. ${ }^{27}$ have stated that they must result from the fact that once a macromolecule (or ol igomer) reaches a certain size, it proceeds in one of two directions from which there is no return. The formation of a proportion of the cyclic oligomers (region A) is explicable in such a way (the remainder of this fraction is formed during polymer degradation by backbiting, vide infra). However, of the polymer in region $B$, consistent with the model of Figure 8, they were only able to observe that "polysilylene chains after growth to the polymerization degree of $\approx 50$ either depart to solution or stick to the sodium surface and grow by reaction with monomer (or with other $\mathrm{Si}-\mathrm{Cl}$ end groups)". Although they showed, just as we have here, that the proportion of this fraction was dependent upon solvent and temperature, its origins have remained an enigma. A significant part of the enigma is that it is difficult to rationalize what is a very consistent degree of polymerization for fraction B simply in terms of polymer solubility effects. In our experience this fraction is found in every system at a peak molecular weight of about 4000-5000 regardless of solvent, temperature of reaction, additives, and degree or method of agitation. A part from cyclic oligomers, it is the only fraction found when homogeneous reducing agents such as sodium naphthalide ${ }^{38}$ or the $\mathrm{K}^{+} / \mathrm{K}^{-} / 18$-crown- 6 complex ${ }^{39}$ are used to mediate the synthesis in THF solution at temperatures as low as $-79{ }^{\circ} \mathrm{C}$, and it is also found in the solution phase when the graphite intercalate, $\mathrm{C}_{8} \mathrm{~K}$, is used as the reducing agent. ${ }^{43,44} \mathrm{It}$ is formed when higher molecular weight PMPS is reacted, typically with Friedel and Craft catalysts, under mild conditions. ${ }^{45-47}$ Similar examples can be cited for other polysilylenes; ${ }^{1}$ in fact the appearance of this mol ecular weight fraction is not merely commonplace, it is ubiquitous.

An al ternative to the view that the polymer molecules of this intermediate molecular weight fraction, B, leave the alkali metal surface and enter solution at some inexplicably definite point during their growth which corresponds to a degree of polymerization of about 50 is the possibility that they derive from high molecular 


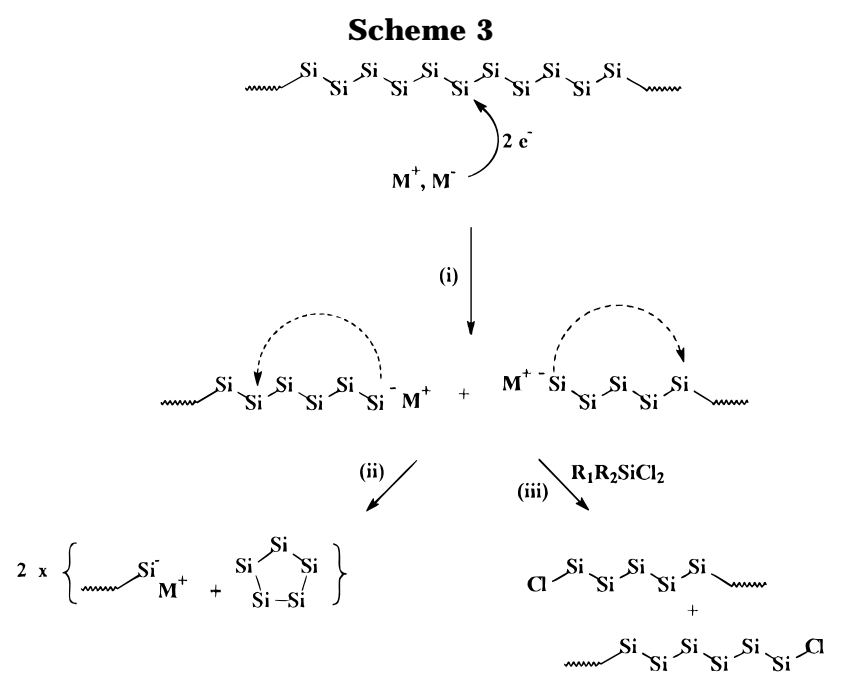

weight polymer following a nonrandom chain scission reaction. Figure 7 supports this possibility for it is clear that the high proportion of high molecular weight polymer that is evidently formed in the early stages of the reaction in toluene is replaced by fraction $B$ as reaction proceeds. ${ }^{48}$ It has always been assumed that the end product of polysilylene degradation is cyclic oligomer arising from the backbiting reaction depicted within Scheme 3 as reaction $\mathrm{i}$, but from the plots of Figure 7 it is clear that the proportion of cyclic oligomer falls slightly while the high molecular weight polymer is apparently undergoing degradation. I ts concentration then rises marginally but the subsequent fluctuations indicate that this is at the expense of the intermediate molecular weight fraction that is found in the solution phase and not at the further expense of the high molecular weight fraction.

A variation of the initiation of the backbiting reaction as represented by Matyjaszewski et al. ${ }^{27}$ is shown in Scheme 3. It is important to recognize that at ambient temperature the complete degradation of a solution of a preformed PMPS in THF can be induced by the addition of sodium naphthalide ${ }^{27}$ while at reduced temperatures it is our observation that the same reagent can be used effectively as the reductive-coupling agent in polymer formation. ${ }^{38}$ Thus the efficiency of the backbiting reaction relative to the polymer forming reaction is significantly reduced by lowering the temperature. The effect of the electron acceptor in such systems is therefore twofold. By solubilizing the sodium it both activates it for the purposes of mediating the polymerization and yet it also acts as a phase transfer catalyst for transporting it to preformed polymer chains where it initiates their degradation. Even at low temperatures, if a stoichiometric excess of sodium naphthalide is used in the synthesis, much less polymer is formed, for the excess initiates the backbiting reaction. ${ }^{38}$ Similar behavior is observed for syntheses mediated by the $\mathrm{K}^{+} / \mathrm{K}^{-} / 18$-crown- 6 system in $\mathrm{THF}^{39}$ and the explanation is essentially the same.

The effects of 15-crown-5 in the various polymerizing systems can now be rationalized, for like an electron acceptor such as naphthalene the crown ether is an effective phase transfer catalyst for sodium. When toluene or other high boiling hydrocarbons at their boiling points are the sustaining solvents in the Wurtz reductive-coupling reaction, the alkali metal is in a molten state and its activation for the purposes of initiating the reaction is thermal. A possible schematic of the overall polymerization reaction by Matyjaszewski et al. ${ }^{27}$ is shown in Figure 9 . When the temperature of

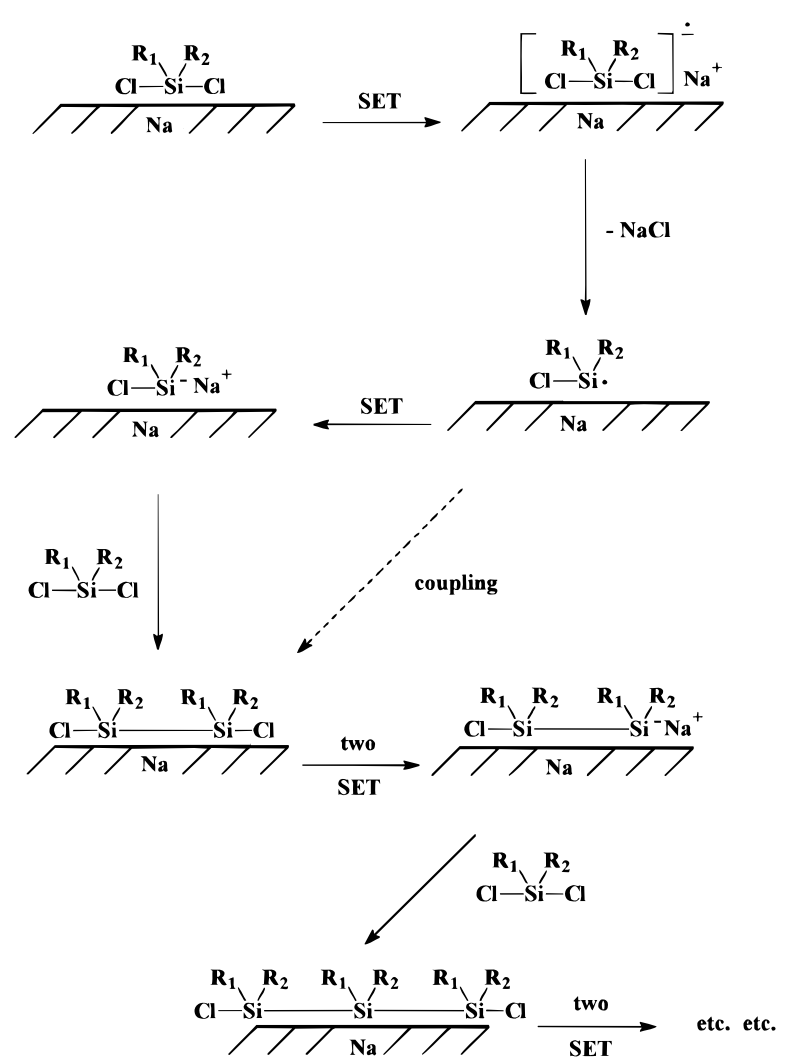

Figure 9. Schematic of the formation of a polysilylene by the thermally activated Wurtz reductive-coupling reaction in accordance with that of Matyjaszewski et al. ${ }^{27}$

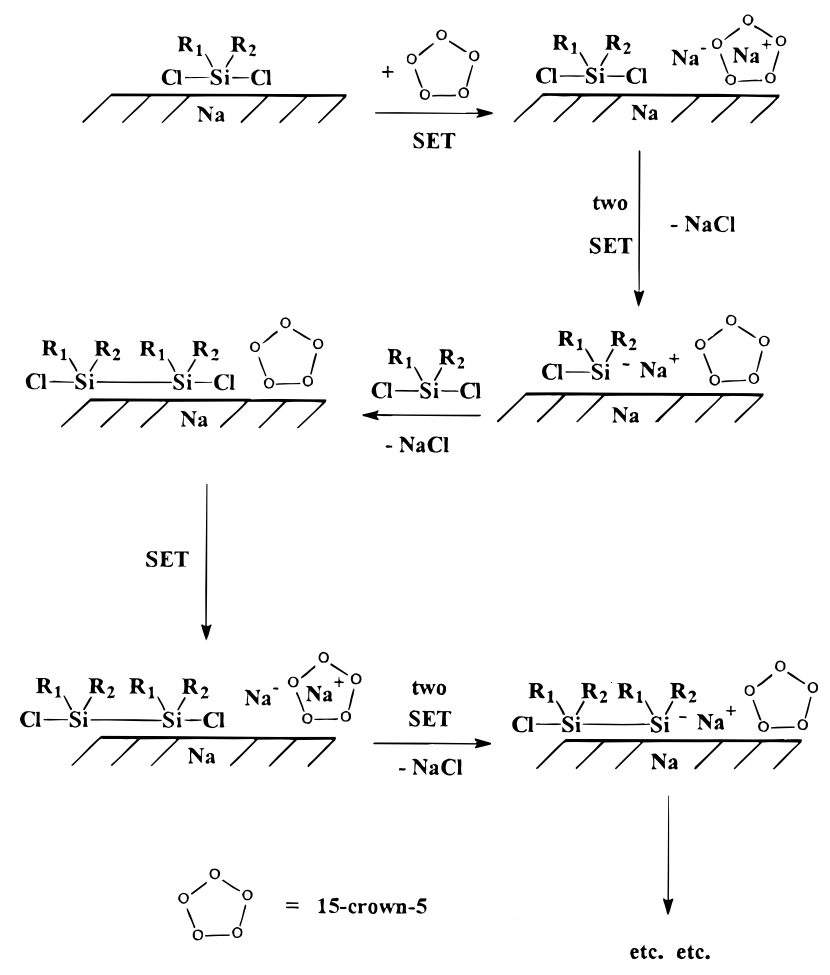

Figure 10. Schematic of the formation of a polysilylene by the Wurtz reductive-coupling reaction activated by a phase transfer catalyst such as 15-crown-5.

reaction is lower, particularly when it is less than the melting point of the alkali metal, thermal activation is significantly reduced but a phase transfer catalyst can then fulfill this role as depicted in Figure 10. Much evidence to support this view can be found in Table 9 for the crown ether enhances the yields of PMPS for the reactions in toluene, xylene, and diethyl ether at temperatures less than the melting point of sodium. Fig- 
ures 2 and 4 for the diethyl ether solvent systems give full representation to the effects of such phase transfer activation. At low concentrations of the crown ether the polymer yields are greatly increased as are the breadths of the molecular weight distributions. Nonetheless, the shoulder on the low molecular weight side of the distribution of Figure $2 \mathrm{~b}$ is evidence that the region $\mathrm{B}$ polymer is still present in the product.

Figures 1 and 3 demonstrate the effect of crown ether when there is al ready adequate thermal activation. At the high temperature of boiling toluene, the rate of chain scission is so high that the addition of even small amounts of the phase transfer catalyst results in the complete destruction of the high molecular weight polymer of region $C$. Though there is not a distinct region $\mathrm{C}$ for the polymer formed at much lower temperatures in the diethyl ether solvent system, it is clear from Figure 4 that even in this case a similar effect can be achieved by adding a considerable excess of the crown ether. In these situations the crown ether is fulfilling its other role and transporting the alkali metal as $\mathrm{Na}^{+} /$ $\mathrm{Na}^{-49,50}$ to sites on preformed polymer chains where it can induce chain scission in accordance with reaction $\mathrm{i}$ of Scheme 3. While ever there is DCMPS (or other mobile $\mathrm{Si}-\mathrm{Cl}$ end groups) still present in the system, the anionic chain ends that are the immediate products of the scission reaction are promptly end-capped in accordance with reaction iii of Scheme 3, and backbiting does not ensue. However, as has previously been noted, 27,29 if the reaction conditions are sustained when crown ether is present, eventual degradation to cyclic oligomers is inevitable and rapid. This does not occur in the reaction as it is usually carried out in a boiling hydrocarbon in the absence of crown ether for the simple reason that there is no mechanism to transport the alkali metal into solution and thence to sites on pre formed polymer chains where it may induce degradation after the remaining DCMPS has been consumed. What degradation of high molecular weight polymer that does occur in the absence of crown ether can only arise as a consequence of adventitious contact of polymer chains with the alkali metal surface during the polymerization process. DCMPS is still present so this contact only results in isolated scissions and the conversion of polymer from region $C$ into the intermediate molecular weight fraction of region $B$ in the general distribution.

The above arguments in which the polymer forming reaction and a chain scission reaction are in competition lend a foundation for understanding all of the results presented here. Both of these reactions will be influenced by temperature and solvent nature, and their relative rates and therefore polymer yields and, where relevant, the proportions of low to high molecular weight polymer $\left(\mathrm{L}_{\mathrm{p}} / \mathrm{H}_{\mathrm{p}}\right)$ that can be isolated will change accordingly. More polar solvents, which stabilize the chain carrying silyl anions of the polymer forming reaction, will also more effectively solvate the charge transfer species that arise from the interaction of the alkali metal with 15-crown-5. To an extent they might act as phase transfer agents in their own right. THF is a case in point for it is clear from Table 9 that, consistent with there al ready being adequate activation, the addition of a small amount of crown ether to this solvent system generally results in a reduction of yields and a narrowing of the molecular weight distributions at all temperatures. It must be assumed that diphenyl ether is so effective at solvating charge transfer species that polymer formation is completely inhibited when the crown ether is added to this system except at the lowest temperatures of reaction.
While to date it has proved impossible to advance a rationale for an apparently nonrandom event interrupting the polymer growth reaction and so leading to the formation of the intermediate molecular weight fraction, there is an explanation for a seemingly nonrandom scission in chains of high molecular weight polymer. Light scattering measurements have demonstrated that polysilylenes in solution are substantially more extended that carbon chain polymers. ${ }^{51}$ Spectroscopic determinations and theoretical considerations ${ }^{52,53}$ have shown them to be characterized by extended sequences of repeat units in approximately all-trans conformation. In the case of PMPS, the average length of these sequences has been estimated to involve from 35 to 50 monomer units, 54 a range which is in excellent correspondence with the observed range of degree of polymerization of the intermediate molecular weight fraction in the product distributions. Such sequences end at strong turns (such as gauche) and these would constitute defects where the continuity of the $\sigma$-delocalization that characterizes the polysilylene chain would be interrupted. Thus, in a long polymer chain a gauche defect represents a point which separates the HOMO of one trans sequence from that of the next. Accordingly, the Si-Si bond that is central to a gauche conformer in the chain is marginally weaker than the bonds in the sequences to either side. Chain scission is initiated by the transfer of el ectrons into the LUMO of the $\sigma$-catenated system but within this orbital they are free to move unhindered by any conformational irregularities in the chain ${ }^{1}$. Thus, they are able to seek out the weakest points in the chains, the gauche defects, and it is at these that their effect would be manifest; i.e., scission would occur selectively at the gauche defects.

The effects of the addition of varying amounts of 15crown-5 to the sodium-mediated reductive coupling of dichloromethyl phenylsilane in a range of hydrocarbon and ether solvents and at various temperatures have thus been shown to correlate with competing mechanisms of polymer formation and a highly selective chain scission reaction. The latter is the precursor to polymer degradation by the generally accepted chain backbiting reaction which under suitable conditions will inevitably ensue. When there is appropriate activation, which might be either thermal or through the intermediacy of a phase transfer agent such as crown ether, the formation of high molecular weight polysilylene corresponding to degrees of polymerization in the region of 50000 is kinetically driven. However, the occurrence of chain scissions that occur continuously throughout the synthesis reaction results in a reduction of the molecular weight of most of the polymer to a much lower value corresponding to a degree of polymerization of about 50. The degradation of polysilylenes to stable cyclic oligomers is thermodynamically driven but polymer at this intermediate degree of polymerization must represent a metastable stage in the overall process. The selective chain scission has been rationalized as occurring at the gauche defects that are statistically distributed among the otherwise extended trans sequences in the catenated silicon backbone of the polymer. Most polysilylenes have molecular weight distributions that display the intermediate molecular weight fraction that has been focal to this investigation and the average molecular weight of this fraction varies from polymer to polymer. To lend further weight to our hypothesis, investigations are now underway to establish a correlation between these and the conformations of a range of polysilylenes in solution. 
Acknowledgment. This work was performed under the management of the J apan High Polymer Centre as a part of the Industrial Science and Technol ogy F rontier Programme supported by the New Energy and Industrial Technology Development Organisation. We also gratefully acknowledge the European Union for the award of a Human Capital and Mobility Fel lowship for U.B., and the United Kingdom Engineering and Physical Sciences Research Council for a postdoctoral fellowship in support of S.J .H. Finally, in acknowledgment of the value of discussion we thank Professor Hideki Sakurai and our colleague Dr. Michael Went.

\section{References and Notes}

(1) Miller, R. D.; Michel, J. Chem. Rev. 1989, 89, 1359 and references therein.

(2) Kipping, F. J . Chem. Soc. 1921, 119, 830.

(3) Kipping, F.J. . Chem. Soc. 1924, 125, 2291.

(4) Trujillo, R. E. J . Organomet. Chem. 1980, 198, C27.

(5) Trefonas III, P. T.; Djurovich, P. I.; Zhang, X.-H.; West, R.; Miller, R. D.; Hofer, D. J . Polym. Sci. Polym. Lett. Ed. 1983, 21, 819.

(6) Wesson, J . P.; Williams, T. C. J . Polym. Sci. Polym. Lett. Ed. 1980, 18, 959.

(7) West, R.; David, L. D.; Djurovich, P. I.; Stearley, K. L.; Srinivasan, K. S. V.; Yu, H. 1981, 103, 7352.

(8) Mark, J. E.; Allcock, H. R.; West, R. Inorganic Polymers; Englewood Cliffs, Prentice-Hall Inc.: NJ , 1992; pp 186-236 and references therein.

(9) Gauthier, S.; Worsfold, D. Macromolecules 1989, 22, 2213.

(10) Miller, R. D.; Rabold, J .; Sooriyakumaran, R.; Flemming, W.; Fickes, G. N.; Farmer, B. L.; Kuzmany, H. In Inorganic and Organometallic Polymers; ACS Symposium Series 360; Zeldin, M., Wynne, K. H., Allcock, H. R., Eds.; American Chemical Society: Washington, DC, 1988; Chapter 4.

(11) West, R.; Wolff, A. R.; Peterson, D. J .J . Radiat. Curing 1986, $13,35$.

(12) Wolff, A. R.; West, R. Appl. Organomet. Chem. 1987, 1, 7.

(13) Yajima, S.; Hasegawa, Y.; Hayashi, J .; I omora, M. J . Mater. Sci. 1980, 15, 720.

(14) Yajima, S.; Hayashi, J .; Omori, M. Chem. Lett. 1975, 931.

(15) Kepler, R. G.;Zeigler, J. M.; Harrah, L. A.; Kurtz, S. R. Bull. Am. Phys. Soc. 1983, 28, 362.

(16) Lovinger, A. J .; Davis, D. D.; Schilling, F. C.; Bovey, F. A.; Zeigler, J . M. Polym. Commun. 1989, 30, 356.

(17) Tilley, T. D. Acc. Chem. Res. 1993, 26, 22.

(18) Sakamoto, K.; Obata, K.; Hirata, H.; Nakajima, M.; Sakurai, H. J. Am. Chem. Soc. 1989, 111, 7641.

(19) Matyjaszewski, K.; Gupta, Y.; Cypryk, M. J . Am. Chem. Soc. 1991, 113, 1046.

(20) Miller, R. D.; Hofer, D.; McKean, D. R.; Willson, C. G.; West, R.; Trefonas III, P. T. In Materials for Microlithography; ACS Symposium Series 266; Thompson, L. F., Willson, C. G., Frechet, J. M. J ., Eds.; American Chemical Society: Washington, DC, 1984; Chapter 14.

(21) Zeigler, J. M. Polym. Prepr. (Am. Chem. Soc., Div. Polym. Chem.) 1986, 27, 109.

(22) Cragg, R. H.; J ones, R. G.; Swain, A. C.; Webb, S. J . J . Chem. Soc., Chem. Commun. 1990, 1147.

(23) Chen, S. M.; David, L. D.; Haller, K. J .; Wadsworth, C. L.; West, R. Organometallics 1983, 2, 409.

(24) Zeigler, J. M.; McLoughlin, L. I.; Perry, R. J. J . Inorg. Organomet. Polym. 1991, 1, 531.

(25) J ones, R. G.; Benfield, R. E.; Cragg R. H.; Swain, A. C. J . Chem. Soc., Chem. Commun. 1992, 1022.

(26) Worsfold, D. J . In Inorganic and Organometallic Polymers; ACS Symposium Series 360; Zeldin, M., Wynne, K. H.,
Allcock, H. R., Eds.; American Chemical Society: Washington, DC, 1988; Chapters 8 and 9.

(27) Matyjaszewski, K.; Greszta, D.; Hrkach, J. S.; Kim, H. K. Macromolecules 1995, 28, 59.

(28) Miller, R. D.; Ginsburg, E.J .; Thompson, D. Polym. J . 1993, $25,807$.

(29) J ones, R. G.; Benfield, R. E.; Cragg, R. H.; Swain, A. C.; Webb, S. J. Macromol ecules 1993, 26, 4878.

(30) Fujino, M.; Isaka, H. J . Chem. Soc., Chem. Commun. 1989, 466.

(31) Matyjaszewski, K. Polym. Prepr. (Am. Chem. Soc., Div. Polym. Chem.) 1987, 28, 224.

(32) Zeigler, J. M.; Harrah, L. A.; J ohnson, A. W. Polym. Prepr. (Am. Chem. Soc., Div. Polym. Chem.) 1987, 28, 424.

(33) J ones, R. G.; Benfield, R. E.; Cragg, R. H.; Swain, A. C. J . Chem. Soc., Chem. Commun. 1992, 112.

(34) Benfield, R. E.; Cragg, R. H.; J ones, R. G.; Swain, A. C. Nature 1991, 353, 340.

(35) Kim, H. K.; Matyjaszewski, K. J . Am. Chem. Soc. 1988, 110, 3321 .

(36) Miller, R. D.; Thompson, D.; Sooriyakumaran, R.; Fickes, G. N. J. Polym. Sci.: Part A: Polym. Chem. 1991, 29, 813.

(37) J enkner, P. K.; Suzuki, H.; Miller, R. D. Polym. Prepr. (Am. Chem. Soc., Div. Polym. Chem.) 1994, 35, 500.

(38) J ones, R. G.; Benfield, R. E.; Cragg, R. H.; Evans P. J .; Swain, A. C. Polym. Commun. 1994, 35, 4924.

(39) Lacave-Goffin, B.; Hevesi, L.; Devaux, J .J . Chem. Soc., Chem. Commun. 1996, 765.

(40) This reaction time was chosen as the standard reaction time for purposes of comparability between the different systems. In some cases, optimum yields or molecular weight parameters were established at shorter reaction times but we have no evidence of there being subsequent substantial polymer degradation unless reactions were sustained for far longer times.

(41) The boiling point of diphenyl ether, $259^{\circ} \mathrm{C}$, is so high as to be considered an unrealistic reaction temperature and even at the temperature chosen for this reaction it was thought prudent to reduce the reaction time.

(42) Wolff, A. R.; Nozue, I.; Maxka, J .; West, R. J . Polym. Sci., Polym. Chem. 1988, 26, 701.

(43) J ones, R. G.; Benfield, R. E.; Evans, P. J .; Swain, A. C. J . Chem. Soc., Chem. Commun. 1995, 1465.

(44) Lacave-Goffin, B.; Hevesi, L.; Devaux, J . J . Chem. Soc., Chem. Commun. 1995, 769

(45) J ones, R. G.; Benfield, R. E.; Cragg, R. H.; Swain, A. C.; Webb S. J .; Went, M. J . Polymer 1995, 36, 393.

(46) J ones, R. G.; Swain, A. C.; Holder, S. J .; Benfield, R. E. In Silicon-containing Polymers; Special Publication No. 166; J ones, R. G., Ed.; The Royal Society of Chemistry: Cambridge, UK, 1995; p 132.

(47) J ones, R. G.; Swain, A. C.; Holder, S.J .; Benfield, R. E.; Went, M. J .; Wiseman, A. J I In Metal Containing Polymeric Materials; Plenum Publishing Corp.: New York, 1996; pp 161-175.

(48) Though this observation only applies to the polymer that is found within the solution phase there is no reason to believe that the situation is appreciably different for polymer that might have precipitated, except perhaps in extent.

(49) Dye, J . L.; Andrews, C. W.; Mathews, S. E. J . Phys. Chem. 1975, 79, 3065

(50) Dye, J. L. Angew. Chem., Int. Ed. Engl. 1979, 18, 587.

(51) Cotts, P. M.; Miller, R. D.; Trefonas III, P. T.; West, R.; Fickes, G. N. Macromolecules 1987, 20, 1046.

(52) Klingensmith, K. A.; Downing, J . W.; Miller, R. D.; Michl, J . . Am. Chem. Soc. 1986, 108, 7438.

(53) Takeda, K.; Teramae, H.; Matsumoto, N. J . Am. Chem. Soc. 1986, 108, 8186

(54) Strazielle, C.; de Mahieu, A.-F.; Daoust, D.; Devaux, J . Polymer 1992, 33, 4174.

MA960496Y 